\title{
Acanthamoebic keratitis diagnosed by paracentesis and biopsy and treated with propamidine
}

\author{
K MCCLELLAN AND D J COSTER \\ From the Department of Ophthalmology, Flinders Medical Centre, Adelaide, South Australia
}

SUMMARY A previously healthy 53-year-old man had keratitis of the right eye for six months, unresponsive to topical medical therapy. Acanthamoeba was grown from tissue obtained by corneal biopsy and from aqueous from an anterior chamber tap. The patient was treated with propamidine isethionate $0.1 \%$ drops and dibromopropamidine isethionate $0.15 \%$ ointment, and after two and a half months the ocular inflammation was continuing to resolve. This case supports a role for the diamidines in the treatment of acanthamoebic keratitis.

Acanthamoebic keratitis is a serious sight threatening condition. It can be difficult to diagnose and very difficult to treat. Despite an increasing number of reports ophthalmologists must base their knowledge of the condition on anecdotal reports of those cases which provide insight into the clinical problem. This report is of a man who had a persistent hypopyon from which the organism was recovered, and who responded to topical administration of propamidine isethionate and dibromopropamidine isethionate.

Acanthamoeba species have now been reported many times as having caused protracted keratitis unresponsive to medical therapy. ${ }^{1-11}$ Reports of successful medical treatment are few. Of these, one has been of treatment with a combination of propamidine isethionate drops $0.1 \%$, dibromopropamidine isethionate $0 \cdot 15 \%$ ointment, and neomycin drops $^{12}$ and another of treatment with topical miconazole $10 \mathrm{mg} / \mathrm{ml}$, polymyxin-neomycingramicidin, and propamidine isethionate. ${ }^{13}$ The case reported here supports a role for diamidines in the medical management of acanthamoebic keratitis.

\section{Case report}

A healthy 53-year-old man was referred with right keratitis, unresponsive to treatment with topical prednisolone acetate $1 \%$ and acyclovir during the preceding month. He remembered no episodes of ocular trauma but had had an attack of keratitis four years previously which healed within two weeks of beginning topical antiviral therapy.

Correspondence to Professor D J Coster. Department of Ophthalmology, Flinders Medical Centre, Bedford Park. South Australia 5042 .
On examination, visual acuity in the right eye was hand movements at $1 \mathrm{~m}$, in the left eye $6 / 5$. In the right eye there was a large central corneal epithelial defect overlying an area of stromal inflammation which extended to the corneal mid-periphery, where the opacification was denser (Fig. 1). Iris details could not be seen. Corneal scrapings grew Staphylococcus epidermidis, and topical treatment was continued with vidarabine ointment, prednisolone sodium phosphate $0 \cdot 5 \%$, and atropine $1 \%$ drops.

After five weeks the anterior segment inflammation had increased and a hypopyon was evident (Fig. 2). On several occasions during the next two months cultures for bacteria, fungi, and amoebae were negative, and the hypopyon persisted, accompanied by severe ocular pain. At the end of this time the

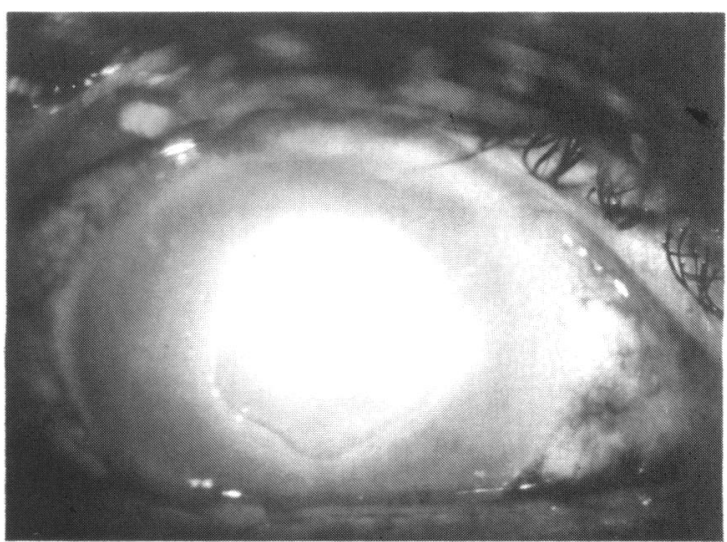

Fig. 1 Large central corneal epithelial defect overlying an area of stromal inflammation. 


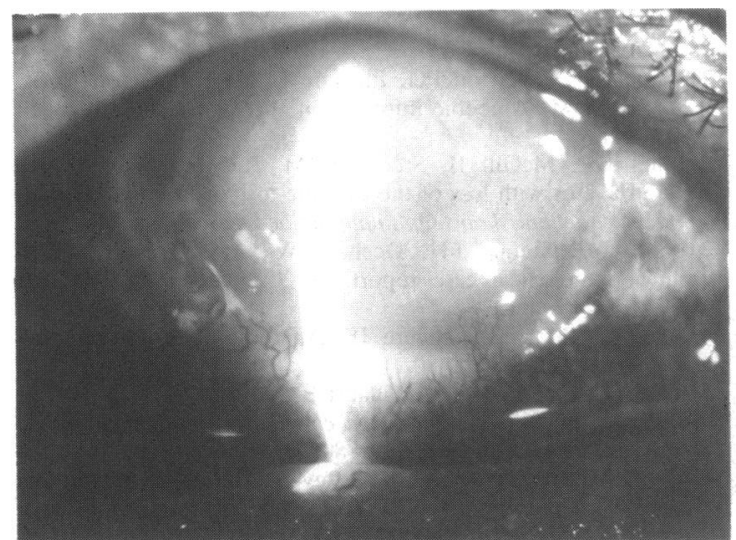

Fig. 2 Central keratitis with hypopyon.

corneal opacity was dense, but the corneal epithelial defect had begun to heal and was completely epithelialised three months after the patient's initial presentation.

For the next two months the condition remained unchanged. At the end of this time aqueous from an anterior chamber tap showed unusual eosinophils but no causative organism. One week later, and six months after the onset of the keratitis, corneal biopsy was performed, with repeat anterior chamber tap. Acanthamoeba grew from both specimens after five days, though none was seen in the histopathological sections of the corneal biopsy. Morphologically, the organism was similar to but not identical with Acanthamoeba polyphaga.

Because of the extent of anterior segment inflammation and the presence of amoebae in the aqueous humour, medical treatment was preferred to surgical excision and corneal grafting. Treatment was started with propamidine isethionate $0.1 \%$ drops two hourly, dibromopropamidine isethionate $0.15 \%$ ointment at night, prednisolone acetate $1 \%$ drops every two hours, and atropine $1 \%$ drops twice daily.

After three weeks there were signs of improvement, with less anterior segment inflammation, disappearance of the hypopyon, and clearing of the stromal infiltrate and oedema, allowing some view of the iris details. This improvement was maintained during the next month; but, with recurrence of a central epithelial defect (Fig. 3), treatment was reduced to propamidine isethionate (). $1 \%$ drops and prednisolone acetate 1\% drops, each four times a day, and atropine $1 \%$ drops twice daily. The eye was padded. The epithelial defect healed within one week, but further reduction of propamidine isethionate $0.1 \%$ to twice daily resulted in a rapid increase in anterior segment inflammation.

Subsequently the patient received propamidine isethionate $0.1 \%$ drops four times a day and dibro-

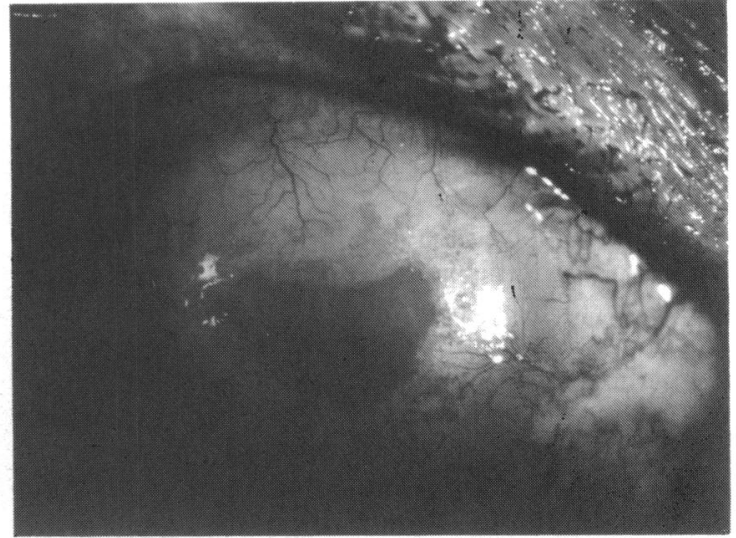

Fig. 3 Central epithelial defect stained with rose Bengal.

mopropamidine isethionate ()$\cdot 15 \%$ ointment at night for two months, then the drops only for a similar period. Further reduction of treatment resulted in increased inflammation, but this tendency decreased with time and ocular inflammation continued to resolve. The eye is now quite comfortable, but visual acuity remains hand movements at $1 \mathrm{~m}$, owing to corneal opacity and cataract (Fig. 4).

\section{Discussion}

Two aspects of this case are unusual. Firstly, acanthamoeba was recovered from the anterior chamber and disaggregated corneal material but could not be seen in a biopsy conducted by personnel experienced in identifying the organism. Secondly, a favourable response to medical therapy was apparent. In other respects the case represents a common clinical problem: a clinical picture at presentation assumed due to herpes simplex virus and an

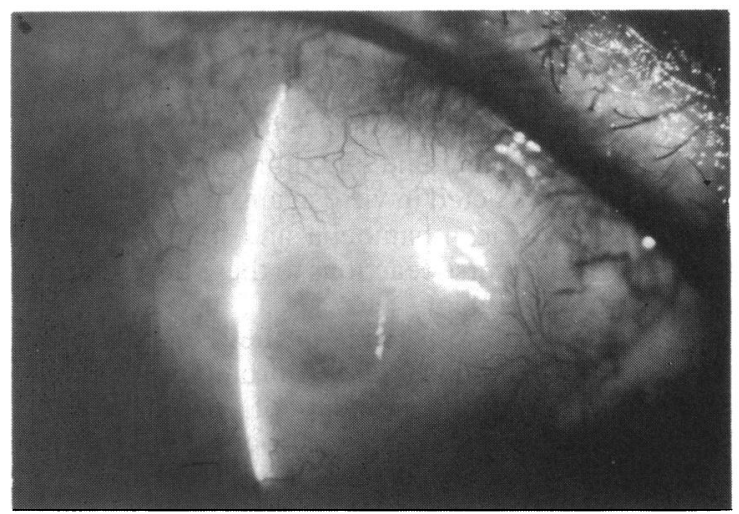

Fig. 4 Appearance of the cornea six months after commencement of medical therapy for acanthamoebic keratitis. The epithelium has healed, but extensive stromal opacity and vascularisation remain. 
encouraging healing of an epithelial defect in response to topical steroids, but with stromal inflammation continuing despite further topical medication. In this situation corneal biopsy needs to be considered, and the results can be most helpful, as in this case.

Acanthamoeba is a small, free-living organism commonly found in soil, fresh water, and sea water, and cysts can occur in airborne dust. Of all free-living amoebae, only acanthamoeba and Naegleria fowleri are pathogenic in man. ${ }^{1+15}$ Two forms of the organism exist: cysts and trophozoites. The cysts are remarkably resistant to desiccation and heat, as well as to chemical disinfectants, but are killed by autoclaving or gas sterilisation. ${ }^{16}$ Both forms of the organism are also resistant to most antimicrobial agents, ${ }^{17-19}$ but cysts are more resistant than trophozoites and may persist in the cornea indefinitely, requiring prolonged topical therapy." ${ }^{21}$

Although acanthamoebic keratitis is reported with increasing frequency, reports are anecdotal, and the outcome is often poor. There have been few indications as to what treatment might be expected to be effective, but the most promising has been with propamidine and dibromopropamidine isethionate.

Propamidine isethionate and dibromopropamidine isethionate are members of the diamidine group of drugs. They both have antibacterial and antifungal activity and have long been used for the treatment of blepharitis and conjunctivitis. Dibromopropamidine isethionate is available as a $0 \cdot 15 \%$ ointment and is reported to be active against pyogenic cocci, Staphylococcus aureus, and Gram-negative organisms, including some strains of Pseudomonas. ${ }^{12}$ Propamidine isethionate is available as $0 \cdot 1 \%$ drops and has a reduced spectrum of antibacterial activity, being active against Gram-positive organisms, including staphylocci and Streptococcus pyogenes, but not against Gram-negative organisms. ."

The diamidines are also toxic to a number of different protozoa, but their mechanism of action has not yet been established." ${ }^{21}$ Wright and colleagues ${ }^{12}$ showed that propamidine and dibromopropamidine isethionate have acted in vitro against both cysts and trophozoites of acanthamoeba and that, clinically, there were no adverse reactions to these drugs during two months' treatment.

The case reported here further supports the place of propamidine and dibromopropamidine isethionate in the treatment of acanthamoebic keratitis and also emphasises the useful role of corneal biopsy in the diagnosis of this condition.

We gratefully acknowledge the assistance of Peter Christy and Bret Robinson of the State Water Laboratories, South Australian Department of Engineering and Water Supply, in identifying acanthamocba.

\section{References}

1 Nagington J, Watson PG, Playfair TJ, McGill J, Jones BR, Stecle ADM. Amoebic infection of the eye. Lancet 1974; ii: 1537-40.

2 Jones BR. McGill JI. Stecle ADM. Recurrent suppurative keratouveitis with loss of the eye due to infection by Acanthamoeba castellani. Trans Ophthalmol Soc UK 1975; 95: 210.

3 Lund OE, Stefani FH, Dechant W. Amocbic keratitis: a clinicopathological case report. Br J Ophthalmol 1978; 62: 373-5.

4 Hamburg A, DeJonckhecre JF. Amoebic keratitis. Ophthalmologica 1980; 181: 74-80.

5 Key SN III, Green WR, Willacrt E, Stevens AR, Key SN Jr. Keratitis duc to Acanthamoeba castellani; a clinicopathologic case report. Arch Ophthalmol 1980; 98: 475-9.

6 Ma P. Willaert E, Juechter KB, Stevens AR. A case of keratitis due to Acanthamoeba in New York, New York, and features of ten cases. J Infect Dis 1981; 143: 662-7.

7 Bos HJ, Völker-Dieben HJM, Kok-van Alphen CC. A case of Acanthamoeba keratitis in The Netherlands. Trans $R$ Soc Trop Med Hyg 1981: 75: 86-91.

8 Samples JK, Binder PS, Laubel FJ, Fort RL, Visvesvara GS, Peter CR. Acanthamoeba keratitis possibly acquired from a hot tub. Arch Ophthalmol 1984; 102: 707-10.

9 Hirst LW, Green WR, Merz W, et al. Management of Acanthamoeba keratitis. A case report and review of the literature. Ophthalmology 1984; 91: 1105-11.

10 Cohen EJ, Buchanan HW, Laughrea PA, et al. Diagnosis and management of Acanthamoeba keratitis. Am J Ophthalmol 1985; 100: 389-95.

11 Roussel TJ, Badenoch PR, Chandraratnam E, Coster DJ. Acanthamocbic keratitis in a healthy Australian man. Med $J$ Aust 1985; 143: 615-7.

12 Wright P. Warhurst D, Jones BR. Acanthamocba keratitis successfully treated medically. $B r$ J Ophthalmol 1985; 69: $778-82$.

13 Moore MR, McCulley JP, Lukenbach M, et al. Acanthamoeba keratitis associated with soft contact lenses. Am J Ophthalmol 1985; 100: 396-403.

14 Visvesvara GS. Free-living pathogenic amoebae. In: Lennette EH, Balows A, Hausler WJ Jr. Traunt JP, eds. Manual of clinical microbiology. Washington, DC: American Society for Microbiology, 1980: 704-8.

15 Warhurst D. Pathogenic frec-living amocbac. Parasitology Today $1985 ; 1: 24-8$.

16 Meisler DM, Rutherford I, Brian FE, et al. Susceptibility of Acanthamoeba to surgical instrument sterilization techniques. Am J Ophthalmol 1985; 99: 724-5.

17 Duma RJ, Finley R. In vitro susceptibility of pathogenic Naegleria and Acanthamoeba species to a variety of therapeutic agents. Antimicrob Agents Chemother 1976; 10: 370-6.

18 Casemore OP. Sensitivity of Hartmannella (Acanthamoeba) to 5-fluorocytosine, hydronystilbamidine and other substances. J Clin Pathol 1970; 23: 649-52.

19 Nagington J, Richards JE. Chemotherapeutic compounds and acanthamoebac from eyc infections. J Clin Pathol 1976; 29: $648-51$

20) Font RL, Tapert MJ, Robinson NM, Visvesvara GS, Murphy D, Osato MS. An animal model of Acanthamoeba keratitis. Further studies with emphasis on the early phase of destruction of trophozoites. Arvo Abstracts. Suppl to Invest Ophthalmol Vis Sci. St Louis: Mosby, 1982: 163.

21 Goodman Gilman A, Goodman LS, Rall TW. Murad F. Goodman and Gilman's The pharmacological basis of therapeutics. New York: McMillan Publishing Company, 1985: $1061-2$.

Accepted for publication 6 October 1986. 